\title{
Sozial-ökologische \\ Transformationsforschung: Desiderata der Kommunikations- und Medienwissenschaft
}

\section{Sigrid Kannengießer}

Keywords: Transformationsforschung, sozial-ökologische Transformation, Nachhaltigkeit, digitale Medien

\section{Abstract}

Auf der Basis zentraler Argumente der interdisziplinären Transformationsforschung im Allgemeinen und der sozial-ökologischen Transformationsforschung im Besonderen sowie des Bereichs der Kommunikations- und Medienwissenschaften, der sich mit Fragen des Wandels beschäftigt, diskutiert dieser Beitrag, wie konkrete Medienpraktiken zur »Großen Transformation «, die auf eine nachhaltige Gesellschaft abzielt, beitragen (können) und wie diese wiederum kommunikations- und medienwissenschaftlich reflektiert werden sollten. Daraus leiten sich Forschungsdesiderata für die Kommunikations- und Medienwissenschaft ab.

Sigrid Kannengießer: Sozial-ökologische Transformationsforschung: Desiderata der Kommunikation- und Medienwissenschaft. In: Nils S. Borchers, Selma Güney, Uwe Krüger und Kerem Schamberger (Hrsg.): Transformation der Medien - Medien der Transformation. Verhandlungen des Netzwerks Kritische Kommunikationswissenschaft. Frankfurt am Main: Westend 2021. DOI: https://doi.org/10.53291/QNC05200.

Prof. Dr. Sigrid Kannengießer I Universität Bremen I sigrid.kannengiesser@ uni-bremen.de 
Dieser Beitrag wurde während der Covid-19-Pandemie geschrieben einer Zeit der globalen Erschütterung etablierter Abläufe. Maskentragen, Abstandhalten und restriktive Begrenzungen der Anzahl der Personen, die sich treffen dürfen, schränken das soziale Leben ein, viele wirtschaftliche Bereiche sind existentiell bedroht, andere, wie der Onlinehandel, wachsen enorm, während die Politik sich (noch mehr als zuvor) an nationalen Fragen und Problemen bei gleichzeitig steigender Notwendigkeit des weltweiten Handelns orientiert.

Während die einen in dieser Krise eine Chance für gesellschaftliche Veränderungen sehen, fürchten andere, dass sie das soziale Gefüge im negativen Sinne stabilisiert: Bestehende Ungleichheiten und Ungerechtigkeiten verschärfen sich innerhalb und zwischen Gesellschaften. Während also die Pandemie wütet, existieren weitere Krisen fort, stehen in interdependenten Verhältnissen und verschärfen sich sogar gegenseitig. So schien die Covid-19-Krise zunächst die sozial-ökologische Krise zu verlangsamen: Kohlenstoffdioxidemissionen und Luftverschmutzung reduzierten sich unter anderem durch die geringere Mobilität im Flug- und individuellen Kraftfahrzeugverkehr. Doch zeigt sich, dass in manchen Bereichen zwar Emissionen eingespart, in anderen dagegen vermehrt produziert werden: Anbieter wie Netflix profitieren von der Pandemie, nicht nur weil die Nachfrage nach ihren Produkten zugenommen hat, sondern auch weil es "zu einer der wenigen Ausspielwege von Hollywood geworden [ist], während die Kinos überall geschlossen sind « (Schuler 2020). Durch die gestiegene Onlinekommunikation in Zeiten der Pandemie, sei es aufgrund der zunehmenden Nachfrage bei Streamingdiensten, der Verlagerung vieler Arbeitsorte ins Home-Office und der damit verbundenen Notwendigkeit zu Videokonferenzen statt Präsenzveranstaltungen und nicht zuletzt des erhöhten Bedarfs nach digitalen Medientechnologien als technischer Voraussetzung, verschärft sich die sozial-ökologische Krise während der Covid-19-Pandemie. Denn nicht nur die materiellen Herstellungsprozesse digitaler Medientechnologien bedürfen Energie, die primär aus fossilen Ressourcen gewonnen wird (Gabrys 2015, 3; Yu et al. 2010). Sie wird auch für die Onlinekommunikation benötigt, hauptsächlich für die riesigen Serverfarmen mitsamt ihren komplexen Kühlanlagen (Cook und Jardmin 2019; Sühlmann-Faul 2019). Bereits vor Covid-19 wurden neun Prozent der global genutzten Energie für das Betreiben digitaler 
Technologien inklusive der benötigten Infrastrukturen verwendet (Morley et al. 2018, 129).

Die »Vielfachkrise« (Demirović et al. 2011) scheint also um eine weitere Krise ergänzt, welche die Weltgesellschaft vor noch größere Probleme stellt. Der Ruf nach einer »Großen Transformation« (WBGU 2011; Schneidewind 2018) ist aktueller, dringlicher und komplexer denn je - gilt es doch vor dem Hintergrund der Pandemie und der sich verschärfenden Ungleichheiten eine komplexe sozio-ökologische Transformation zu forcieren, welche die Krisen mitsamt ihrer Interdependenzen adressiert.

Krüger und Meyen $(2018,343)$ greifen die Forderung nach der Großen Transformation für die Kommunikations- und Medienwissenschaft auf und fordern eine Öffnung des »eingeschränkten Horizonts« des Faches, das in ihren Augen »vor allem für sich selbst arbeitet« (ebd.). Sie schlagen der Disziplin das Ziel einer »Demokratischen Postwachstumsgesellschaft« vor, um »Forschung und Lehre« Sinn zu geben und »schnell den Übergang in eine Postwachstumsgesellschaft zu schaffen und die Demokratie gleichzeitig nicht nur zu erhalten, sondern substanziell zu stärken und auf die Weltgesellschaft auszudehnen« (ebd., 347-348).

Während die Autoren im Anschluss diverse Anforderungen an das Fach skizzieren (ebd., 351-353), um schließlich bei Verteilungsfragen zu landen (ebd., 353-355), folgt dieser Beitrag ihrem Aufruf, sich auch in der Kommunikations- und Medienwissenschaft mit der Großen Transformation zu beschäftigen. Dabei wird diese Forderung auf der Basis der interdisziplinären Transformationsforschung im Allgemeinen und der sozial-ökologischen Transformationsforschung im Besonderen sowie des Bereichs der Kommunikations- und Medienwissenschaften, der sich mit Fragen von Transformation und gesellschaftlichem Wandel beschäftigt, diskutiert. Im Mittelpunkt stehen dabei diejenigen Aspekte der Großen Transformation, die im Sinne einer »nachhaltigen Entwicklung« (WCED 1987) die Interdependenzen sozialer, ökologischer und ökonomischer Gerechtigkeit berücksichtigen.

Des Weiteren wird an Beispielen gezeigt, wie konkrete Medienpraktiken zur Großen Transformation beitragen können und wie diese wiederum kommunikations- und medienwissenschaftlich reflektiert werden sollten. So lassen sich schließlich in diesem Zusammenhang konkrete Desiderata für die Kommunikations- und Medienwissenschaft ableiten. 


\section{Interdisziplinäre Transformationsforschung und kommunikations- und medienwissenschaftliche Theorien des Wandels}

Um kommunikations- und medienwissenschaftliche Desiderata zu entwickeln, welche die Frage nach der Großen Transformation adressieren, wird im Folgenden zunächst die Transformationsforschung im Allgemeinen und insbesondere die sozio-ökologische Transformationsforschung im Besonderen definiert. Zusätzlich soll gezeigt werden, wie sich die Kommunikations- und Medienwissenschaft bislang mit gesellschaftlichem Wandel beschäftigt hat.

\subsection{Interdisziplinäre Transformationsforschung}

Transformation ist im wissenschaftlichen Diskurs kein neuer Begriff, ${ }^{1}$ wird aber dennoch als »neues Konzept sozialen Wandels« (Reißig 2009) gehandelt. Mit Hinblick auf die beteiligten Disziplinen, zentralen gesellschaftlichen Bereiche, Themen und Phänomene sowie verwendeten Theorien und Methoden gestaltet sich das Forschungsfeld dabei durchaus komplex. ${ }^{2}$ Transformationsforschung beschäftigt sich nicht mit jeglichem gesellschaftlichen Wandel, sondern mit Prozessen, die »systematischen und substantiellen Charakter tragen, durch identifizierbare Akteure eher revolutionär und steuerungsorientiert begonnen werden sowie deutliche imitative Merkmale aufweisen« (ebd., 12). Der Begriff denotiert also nicht gesellschaftlichen Wandel per se, sondern solche Prozesse, die mit Absicht herbeigeführt werden und deren gesellschaftliche Veränderungen sich über die Zeit hinweg beständig vollziehen. Schien die Relevanz und Brisanz gesellschaftlicher TransformationsprozesseinEuropa nach 1989 abzunehmen, so gewinnen diese-und damit auch die Forschung - vor dem Hintergrund der aktuellen komplexen Krisensituation erneut an politischer und gesellschaftlicher Dringlichkeit.

Aufgrund der ökologischen Krise und des Klimawandels beschäftigt sich die interdisziplinäre Transformationsforschung jüngst vor allem

1 Zur Begriffsgeschichte vgl. Kollmorgen, Merkel und Wagener (2015a, 12-14).

2 Für einen Ein- beziehungsweise Überblick in die Transformationsforschung vgl. Kollmorgen, Merkel und Wagener (2015b). 
mit dem Konzept der Nachhaltigkeit: Ihr Ziel ist es, »Transformation(en) hin zu einer nachhaltigen Gesellschaft zu beschreiben, erklären, bewerten und unterstützen.« (Wittmayer und Hölscher 2017, 11). Im Sinne des Brundtland-Berichts wird eine Entwicklung als nachhaltig charakterisiert, wenn die Bedürfnisse der gegenwärtigen Generationen befriedigt werden, ohne dabei die Bedürfnisse zukünftiger Generationen zu beeinträchtigen (WCED 1987). Die Nachhaltigkeitsforschung hat verschiedene Modelle entwickelt, um den zugrundeliegenden Begriff zu differenzieren, darunter das Drei-Säulen-Modell (Hauff und Claus 2012, 59) und jüngst das Doughnut-Modell (Raworth 2017). Gemein ist ihnen die Betonung, dass Nachhaltigkeit nicht nur eine ökologische, sondern auch eine soziale und ökonomische Dimension umfasst. Als Ziele für nachhaltige Entwicklungen werden genannt: die Sicherung der menschlichen Existenz, die Bewahrung der globalen ökologischen Ressourcen, der Erhalt des gesellschaftlichen Produktivpotenzials und die Gewährleistung der Handlungsmöglichkeiten heutiger und zukünftiger Generationen (Pufé 2014, 18). Die 17 Ziele für nachhaltige Entwicklung, welche die Staatengemeinschaft der Vereinten Nationen 2015 verabschiedete (Vereinte Nationen 2015), konkretisieren diese Ambitionen.

Transformationsforschung, die Nachhaltigkeit in den Fokus setzt, nutzt daher oftmals den Zusatz »sozial-ökologisch«, um zu betonen, dass beide Dimensionen gleichermaßen nötig sind (Brand und Brad 2019). Das Diktum »Transformation ist heute ein Schlüsselbegriff des Nachhaltigkeitsdiskurses« (Luks, Bohunovsky und Höltl 2018, 257) gilt also auch umgekehrt. Während die Transformationsforschung auch die sozial-ökologischen Auswirkungen der Digitalisierung aufgreift (beispielsweise Höfner und Frick 2019; Lange und Santarius 2018), werden sozial-ökologische Prozesse, welche das Ziel der Nachhaltigkeit verfolgen, im Fach der Kommunikations- und Medienwissenschaften kaum thematisiert (Kannengießer 2020a). Doch kommt dem Fach eine zentrale Verantwortung in Hinblick auf Nachhaltigkeit zu (ebd.). Bislang setzt sich die Kommunikations- und Medienwissenschaft durchaus explizit mit Transformationen im Sinne eines gesellschaftlichen Wandels auseinander, jedoch noch zu wenig mit Fragen ihrer sozial-ökologischen Dimension. 


\subsection{Kommunikations- und medienwissenschaftliche Theorien des Wandels}

Vor allem der Wandel von Medien sowie das Zusammenspiel von gesellschaftlichem und medialem Wandel standen bislang im Fokus kommunikations- und medienwissenschaftlicher Forschung, die sich mit Transformation auseinandersetzt.

Kinnebrock, Schwarzenegger und Birkner $(2015,12)$ konstatieren, dass Medienwandel sin den letzten Jahren eines der bestimmenden Schlagwörter im kommunikations- und medienwissenschaftlichen Fachdiskurs geworden « sei. Forschende des Fachs befassten sich mit Wandel in nahezu allen Bereichen des sozialen Lebens, in denen Praktiken und Phänomene der medienvermittelten Kommunikation eine Rolle spielen können (ebd., 11-13.). Auch wenn die Autor*innen als Herausgeber*innen des Sammelbands Theorien des Medienwandels mit diesem Titel den Fokus auf Medien setzen, so betonen sie doch, dass »die Verbindung zwischen Wandel von gesellschaftlich-kulturellen Phänomenen einerseits und sich wandelnden Praktiken der medialen Kommunikation sowie den dafür verfügbaren Kommunikationstechnologien andererseits « (ebd., 12) oftmals in den Blick genommen werden.

So wird der Wandel bezüglich der Medieninhalte (vgl. Donsbach und Büttner 2005) genauso untersucht wie medientechnologische Veränderungen durch Innovationen (vgl. Latzer 2013; Kannengießer 2020c), der Wandel der medialen Öffentlichkeit (vgl. Jarren 1998; Imhof 2011; van Dijk und Poell 2015), des Journalismus (vgl. Lünenborg und Berghofer 2010), des Medienrechts (vgl. Dommann 2014), der Medienindustrie (vgl. Winseck und Jin 2011) sowie das Zusammenspiel des medialen und gesellschaftlichen Wandels (vgl. Münch und Schmidt 2005; Krotz 2007; Hepp, Breiter und Hasebrink 2017). Auch sind die theoretischen Zugänge, die Medienwandel erklären wollen, durchaus komplex und haben eine lange Tradition, wie Wilke (2015) in einer Systematisierung unterschiedlichster Theorieansätze zeigt.

Doch auch wenn verschiedene Ansätze das Zusammenspiel von medialem und gesellschaftlichem Wandel in den Blick nehmen, so offenbart sich tatsächlich, wie Krüger und Meyen (2018) feststellen, eine Forschungslücke in Hinblick auf die Frage nach der Großen Transformation. Dieser Beitrag diskutiert nun im Folgenden, wie sich die Kommunikations- und Medienwissenschaft mit der Großen Transformation beschäftigen kann, indem sie den Fokus auf Medienpraktiken setzt, die zu sozial-ökologischen Transformationsprozessen beitragen wollen. 


\section{Forschungsdesiderata: Medienpraktiken für die Große Transformation}

In ihrem Plädoyer für eine transformative Kommunikationswissenschaft fordern Krüger und Meyen (2018) das Fach auf, sich auf den »Weg in die Postwachstumsgesellschaft« zu machen. Der dahinterliegende ökonomische Ansatz sieht eine "Wirtschaft ohne Wachstum" (vgl. Paech 2005) vor, in der sich die Rolle der Individuen verändert, die nicht mehr »passive« Konsumierende sind und Güter als solche regelmäßig konsumieren und ersetzen, sondern durch einen entsprechenden Umgang mit diesen zu deren Langlebigkeit und schließlich auch zu einer nachhaltigen Gesellschaft beitragen (Paech 2012, 6061). So gilt das Reparieren in diesem Kontext als zentrale Praktik einer nachhaltigen sozialen Ordnung (Nagel und Paech 2018).

Auch in der Kommunikations- und Medienwissenschaft wurde das Reparieren von Medientechnologien als Medienpraktik für eine nachhaltige Gesellschaft diskutiert (vgl. Kannengießer 2017; 2020b). ${ }^{3}$ In empirischen Studien zeigte sich, dass Menschen ihre Medienapparate reparieren, um die Nutzungsdauer der Geräte zu verlängern und damit nicht nur die Produktion von Müll zu vermeiden, sondern auch Ressourcen zu schonen (ebd.). Damit versuchen sie durch die Praktik des Reparierens über eine sozial-ökologische Transformation hin zu einer nachhaltigeren Gesellschaft zu wirken (ebd.).

Dieses Ziel verfolgen auch Akteur*innen, die Medientechnologien unter fairen Bedingungen mit nachhaltigen Ressourcen produzieren oder nutzen. Das wohl prominenteste Beispiel auf dem Markt ist das Fairphone, ein Smartphone des gleichnamigen niederländischen Unternehmens, das unter ebensolchen Bedingungen und Ressourcennutzung hergestellt werden soll. Auch die Produktion und Aneignung dieses Produkts wurde aus kommunikations- und medienwissenschaftlicher Perspektive untersucht (vgl. Kannengießer

3 Eine Praktik ist ein sozial geteiltes, routinisiertes und durch ein implizites sowie interpretatives Wissen gekennzeichnetes, »sozial sverstehbares< Bündel von Aktivitäten « (Reckwitz 2003, 289); Medienpraktiken sind entsprechend auf diese Weise charakterisierbare Handlungen, die sich auf Medien beziehen (für einen Überblick des Forschungsfeldes Gentzel 2015, 15-17; Pentzold 2015). Dabei wird zwischen solchen differenziert, in denen Medien als Vermittler in Kommunikationsprozessen fungieren, und solchen, in denen sie (als Inhalte, Organisationen oder Technologien) selbst im Fokus der Praktiken stehen (Kannengießer und Kubitschko 2017). 
2020b; 2020c). Obwohl im Fairphone nur wenige nachhaltig abgebaute Rohstoffe zu finden sind (Dießenbacher und Reller 2016) und das Unternehmen durch verschiedene Strategien den Neukauf eines Smartphones bewirbt und damit letztendlich doch wieder die Nachfrage ankurbelt und den Ressourcenverbrauch in Kauf nimmt (Kannengießer 2020c), kann die Produktion und Nutzung dieser Medientechnologie als Versuch gesehen werden, zu sozial-ökologischen Transformationsprozessen beizutragen, die auf Nachhaltigkeit zielen (Kannengießer 2020b; 2020c; zu nachhaltigen Medientechnologien auch van der Velden 2018).

Sowohl das Reparieren als auch die Produktion und Nutzung fairer Medientechnologien sind also Beispiele dafür, wie Medienpraktiken zu einer Großen Transformation beitragen können - auch wenn diesen Praktiken Ambivalenzen inhärent sind. So regt das Fairphone-Unternehmen durch Werbestrategien und Kaufanreize doch eher Konsum an, anstatt Nichtkonsum als nachhaltigste Praktik zu proklamieren. Außerdem problematisch ist der Umstand, dass sich solche Praktiken nur in einer gesellschaftlichen Nische vollziehen (Kannengießer 2020b; 2020c). Die handelnden Akteur*innen versuchen mit diesen bewusst, gesellschaftlichen Wandel im Sinne eines engen Begriffsverständnisses von Transformation auszulösen, der nicht nur substantiell, sondern auch beständig wirkt.

Bezweckt man aus kommunikations- und medienwissenschaftlicher Perspektive die gesellschaftlichen Transformationsprozesse, die auf Nachhaltigkeit zielen, zu verstehen und kritisch zu reflektieren, ist es geboten, diese und weitere Medienpraktiken in den Blick zu nehmen. Im Folgenden werden daher anhand einiger Beispiele Forschungsdesiderata entwickelt, anhand deren das Zusammenspiel zwischen den Medien und der Großen Transformation analysiert werden kann. Dabei werden erstere als Technologien, Inhalte und Organisationen begriffen sowie ihre Aneignung berücksichtigt.

In Hinblick auf Medientechnologien gilt es beispielsweise, weitere Initiativen zu analysieren, die Medienapparate unter fairen Bedingungen herstellen (wollen) wie etwa in Deutschland die Computermaus von Nager IT (Kannengießer 2016) oder das Smartphone Shift, das ähnlich dem Fairphone als modulares Gerät reparierbar sein und unter fairen Bedingungen produziert werden soll. Aus einer kommunikations- und medienwissenschaftlichen Perspektive ist es erforderlich, solche Initiativen weitergehend zu untersuchen, um ihr transformatives Potenzial aufzuzeigen und durch die Sichtbarmachung von Grenzen 
und Ambivalenzen dieser Projekte (wie oben am Beispiel des Fairphones skizziert) zu ihrer kritischen Weiterentwicklung beizutragen.

Auch wie Medientechnologien nach dem Ende ihrer Nutzungsdauer zu entsorgen sind, ohne dass sie auf den großen Müllhalden der Welt die Gesundheit von Menschen und Tieren gefährden oder deren Lebensgrundlage zerstören (zu den sozio-ökologische Auswirkungen elektronischen Mülls vgl. Kaitatzi-Whitlock 2015; Gabrys 2011), sollte untersucht werden. Die Verlängerung der Nutzungsdauer durch das bereits thematisierte Reparieren von Medientechnologien stellt dabei zumindest einen Versuch dar, ihre Entsorgung zu verhindern oder zumindest zu verschieben.

Neben der Praktik der Instandsetzung ist es in der Kommunikations- und Medienwissenschaft relevant, weitere Analysen der Medienaneignung vorzunehmen, mit denen Menschen zu einer nachhaltigen Gesellschaft beitragen wollen. Erkenntnisbringend sind etwa Praktiken der Nichtnutzung, die zwar schon im Fokus der Forschung stehen (vgl. Roitsch 2020; Kaun und Schwarzenegger 2014; Portwood-Stacer 2013), aber noch nicht im Zusammenhang mit der Großen Transformation gedacht werden.

Dass die zunehmende Aneignung von Onlinemedien zu einem erhöhten Energiebedarf und -verbrauch führt, wurde eingangs bereits thematisiert. Dieser steigt nicht nur durch die Nutzung der digitalen Endgeräte, sondern auch durch den Betrieb riesiger Serverfarmen, die durch jeden Klick in Onlinemedien aktiviert werden, sowie deren komplexe Kühlanlagen (Gabrys 2015). Problematisch ist hierbei vor allem, dass der Strom derzeit maßgeblich aus fossilen Quellen stammt (ebd.). In diesem Zusammenhang gilt unter anderem festzustellen, inwiefern sich dieser Energieverbrauch und die produzierte Abwärme nachhaltiger nutzen lassen (vgl. Velkova 2016).

Schließlich sollte man auch auf der Inhaltsebene den Zusammenhang von Medien und der Großen Transformation näher in den Blick nehmen. So existieren kaum Studien zu der Frage, wie durch Medieninhalte zu einer nachhaltigen Gesellschaft beigetragen werden kann. ${ }^{4}$ Gerade vor dem Hintergrund der UN-Ziele für nachhaltige Entwicklung, die unterschiedliche gesellschaftliche Transformationsprozesse fordern, ist es von Aktualität und Dringlichkeit (da sie bis 2030 umgesetzt sein sollen), die

4 Für Ausnahmen vgl. Kannengießer (2020c) über die Onlineplattform utopia.de, die für nachhaltigen Konsum wirbt, oder Young und McComas (2016) zu Medien und ihrem Beitrag für eine nachhaltige Entwicklung in Sambia. 
Rolle von Medienorganisationen und ihren Inhalten für die Forderung nach Nachhaltigkeit und der Großen Transformation zu untersuchen.

Mit diesen Beispielen seien einige Forschungsdesiderata der Kommunikations- und Medienwissenschaft aufgezeigt, die im Zusammenhang von Medien und der Großen Transformation bestehen.

\section{Kommunikations- und medienwissenschaftliche Transformationsforschung}

Die aktuelle Transformationsforschung setzt einen Schwerpunkt auf gesellschaftliche Wandlungsprozesse, die auf Nachhaltigkeit zielen. Im Sinne der Großen Transformation (WBGU 2011) werden solche Prozesse analysiert, kritisch reflektiert und in transdisziplinären Ansätzen auch begleitet, die einen gesellschaftlichen Wandel mit dem Ziel der Nachhaltigkeit verfolgen. Obwohl sich, wie an einigen Beispielen illustriert, die Kommunikations- und Medienwissenschaft mit gesellschaftlichem Wandel (vor allem im Zusammenspiel mit medialem Wandel) beschäftigt, gibt es kaum Arbeiten, die sich mit einem oben ausgeführten engeren Fokus gesellschaftlicher Transformationsprozesse, die auf Nachhaltigkeit zielen, auseinandersetzen.

Auf der Grundlage dieser Erkenntnis schließe ich mich der Forderung von Krüger und Meyen (2018) an, dass sich auch die Kommunikationsund Medienwissenschaft mit der Großen Transformation beschäftigen sollte. Eine Möglichkeit, dieser nachzukommen, besteht darin, sich mit konkreten Medienpraktiken auseinanderzusetzen, mit denen Akteur*innen versuchen, auf eine nachhaltige Gesellschaft hinzuwirken. In diesem Beitrag wurden einige Studien vorgestellt, die untersuchen, wie mit Medien (als Inhalte und Technologien) zu einer nachhaltigen Gesellschaft beigetragen werden kann, sowie anhand weiterer Beispiele Forschungsdesiderata für die Kommunikations- und Medienwissenschaft aufgezeigt. Will man die Große Transformation auch aus kommunikations- und medienwissenschaftlicher Perspektive kritisch analysieren und begleiten, so sind diese Lücken zu schließen und gesellschaftliche Herausforderungen und Phänomene weiterhin kritisch zu beobachten, um zusätzliche Desiderata zu identifizieren. Neben diesen Betrachtungen ist es auch eine Aufgabe der Kommunikations- und Medienwissenschaft, in transformativen Projekten Gesellschaft mitzugestalten, etwa durch die Unterstützung nachhaltiger Medienpraktiken oder die Entwicklung nachhaltiger Medientechnologien. Solche 
transformativen Projekte lassen sich dabei vor allem durch transdisziplinäre Ansätze, also in Kooperation mit Akteur*innen der Medienpraxis (Nichtregierungsorganisationen und Unternehmen), umsetzen.

Kommunikations- und Medienwissenschaftler*innen werden damit selbst zu Gestalter*innen der Großen Transformation, die sie gleichzeitig (selbst)kritisch analysieren.

\section{Literatur}

Brand, Ulrich, und Alina Brad. 2019. Sozial-ökologische Transformation. In: Wörterbuch Land- und Rohstoffkonflikte, herausgegeben von Jan Brunner, Anna Dobelmann, Sarah Kirst und Louisa Prause, 279-285. Bielefeld: Transcript.

Cook, Gary, und Elizabeth Jardim. 2019. Clicking Clean Virginia. The Dirty Energy Powering Data Center Alley. Greenpeace Reports. https://www.greenpeace. org/usa/wp-content/uploads/2019/02/Greenpeace-Click-Clean-Virginia-2019. pdf. Zugegriffen: 20. Februar 2019.

Demirovi, Alex, Julia Dück, Florian Becker, und Pauline Bader. 2011. VielfachKrise. Im finanzmarktdominierten Kapitalismus. Hamburg: VSA.

Dießenbacher, Joshua, und Armin Reller. 2016. Das »Fairphone«: Ein Impuls in Richtung nachhaltige Elektronik? In: Kritische Rohstoffe in der Großen Transformation: Metalle, Stoffstrompolitik und Postwachstum, herausgegeben von Andreas Exner, Martin Held und Klaus Kümmerer, 269-292. Wiesbaden: Springer.

Dommann, Monika. 2014. Autoren und Apparate: Die Geschichte des Copyrights im Medienwandel. Frankfurt am Main: S. Fischer.

Donsbach, Wolfang, und Katrin Büttner. 2005. Boulevardisierungstrend in deutschen Fernsehnachrichten. Publizistik 50: 21-38.

Gabrys, Jennifer. 2015. Powering the Digital: From Energy Ecologies to Electronic Environmentalism. In: Media and the Ecological Crisis, herausgegeben von Richard Maxwell, Jon Raundalen und Nina Lager Vestberg, 3-18. Milton Park: Routledge.

Gabrys, Jennifer. 2011. Digital Rubbish: A Natural History of Electronics. Ann Arbor: University of Michigan Press.

Gentzel, Peter. 2015. Praxistheorie und Mediatisierung: Grundlagen, Perspektiven und eine Kulturgeschichte der Mobilkommunikation. Wiesbaden: Springer VS.

Hauff, Michael von, und Katja Claus. 2012. Fair Trade: Ein Konzept nachhaltigen Handels. Stuttgart: UTB.

Hepp, Andreas, Andreas Breiter, und Uwe Hasebrink (Hrsg.). 2017. Commuicative Figurations: Transforming communications in times of deep mediatization. London: Palgrave Macmillan

Höfner, Anja, und Vivian Frick (Hrsg.). 2019. Was Bits und Bäume verbindet: Digitalisierung nachhaltig gestalten. München: Oekom. 
Imhof, Kurt. 2011. Die Krise der Öffentlichkeit: Kommunikation und Medien als Faktoren des sozialen Wandels. Frankfurt am Main: Campus.

Jarren, Otfried. 1998. Medien, Mediensystem und politische Öffentlichkeit im Wandel. In: Politikvermittlung und Demokratie in der Mediengesellschaft: Beiträge zur politischen Kommunikationskultur, herausgegeben von Ulrich Sarcinelli, 74-94. Bonn: Bundeszentrale für politische Bildung.

Kaitatzi-Whitlock, Sophia. 2015. E-waste, Human-waste, Inflation. In: Media and the Ecological Crisis, herausgegeben von Richard Maxwell, Jon Raundalen und Nina Lager Vestberg, 69-84. Milton Park: Routledge.

Kannengießer, Sigrid. 2020a. Nachhaltigkeit und das »gute Leben«: Zur Verantwortung der Kommunikations- und Medienwissenschaft in digitalen Gesellschaften. Publizistik 65 (1): 7-20.

Kannengießer, Sigrid. 2020b. Acting on Media for Sustainability. In: The Turn to Practice in Media Research: Implications for the Study of Citizen- and Social Movement Media, herausgegeben von Hilde Stephansen und Emiliano Treré, 176188. London: Routledge.

Kannengießer, Sigrid. 2020c. Fair Media Technologies: Innovative Media Devices for Social Change. Media Innovations 6 (1): 38-49.

Kannengießer, Sigrid. 2017. »I am not a Consumer Person«: Political Participation in Repair Cafés. In: (Mis)Understanding Political Participation: Digital Practices, New Forms of Participation and the Renewal of Democracy, herausgegeben von Jeffrey Wimmer, Cornelia Wallner, Rainer Winter und Karoline Oelsner, 78-94. London: Routledge.

Kannengießer, Sigrid. 2016. Conceptualizing Consumption-Critical Media Practices as Political Participation. In: Politics, Civil Society and Participation, herausgegeben von Leif Kramp et al., 193-207. Tartu: Tartu University Press.

Kannengießer, Sigrid, und Sebastian Kubitschko. 2017. Editorial. Acting on Media: Influencing, Shaping and (Re)configuring the Fabric of Everyday Life. Media and Communication 5 (3): 1-4.

Kaun, Anne, und Christian Schwarzenegger. (2014). »No Media, Less Life?« Online Disconnection in Mediatized Worlds. First Monday 19 (11). DOI:10.5210/fm. v19i11.5497.

Kollmorgen, Raj, Wolfgang Merkel, und Hans-Jürgen Wagener. 2015a. Transformation und Transformationsforschung: Zur Einführung. In: Handbuch Transformationsforschung, herausgegeben von Raj Kollmorgen, Wolfgang Merkel und HansJürgen Wagener, 11-30. Wiesbaden: Springer VS.

Kollmorgen, Raj, Wolfgang Merkel, und Hans-Jürgen Wagener (Hrsg.). 2015b. Handbuch Transformationsforschung. Wiesbaden: Springer VS.

Kinnebrock, Susanne, Christian Schwarzenegger, und Thomas Birkner. 2015. Theorien des Medienwandels: Konkturen eines emergierenden Forschunsgfeldes? In: Theorien des Medienwandels, herausgegeben von Kinnebrock, Susanne, Christian Schwarzenegger und Thomas Birkner, 11-28. Köln: Herbert von Halem.

Krotz, Friedrich. 2007. Mediatisierung: Fallstudien zum Wandel von Kommunikation. Wiesbaden: Springer VS.

Krüger, Uwe, und Michael Meyen. 2018. Auf dem Weg in die Postwachstumsgesell- 
schaft: Plödoyer für eine transformative Kommunikationswissenschaft. Publizistik 63 (3): 341-357.

Lange, Steffen, und Tilmann Santarius 2018. Smarte grüne Welt? Digitalisierung zwischen Überwachung, Konsum und Nachhaltigkeit. München: Oekom.

Latzer, Michael. 2013. Medienwandel durch Innovation, Ko-Evolution und Komplexität: Ein Aufriss. Medien \& Kommunikationswissenschaft 61 (2): 235-252.

Lünenborg, Margreth, und Simon Berghofer. 2010. Politikjournalismus im Wandel: Merkmale, Einstellungen \& Perspektiven deutscher Politikjournalisten angesichts aktueller Entwicklungen im Berufsfeld. Fachjournalist 10 (3): 17-25.

Luks, Fred, Lisa Bohunovsky, und Andrea Höltl. 2018. Transformationsforschung: Wirtschafts- und sozialwissenschaftliche Perspektiven. GAIA - Ecological Perspectives for Science and Society 27 (2): 257-259.

Münch, Richard, und Jan Schmidt. 2005. Medien und sozialer Wandel. In: Mediensoziologie: Grundlagen und Forschungsfelder, herausgegeben von Michael Jäckl, 201-218. Wiesbaden: VS.

Morley, Janine, Kelly Widdick, und Mike Hazas (2018): Digitalisation, Energy and Data Demand: The Impact of Internet Traffic on Overall and Peak Electricity Consumption. Energy Research \& Social Science 38: 128-137.

Nagel, Manuel, und Niko Paech. 2018. Reparatur kontra Obsoleszenz: Chancen für eine Postwachstumsökonomie. In: Konsumkritische Projekte und Praktiken: Interdisziplinäre Perspektiven auf gemeinschaftlichen Konsum, herausgegeben von Sigrid Kannengießer und Ines Weller, 39-56. München: Oekom.

Paech, Niko. 2005. Nachhaltiges Wirtschaften jenseits von Innovationsorientierung und Wachstum: Eine unternehmensbezogene Transformationstheorie. Marburg: Metropolis.

Paech, Niko. 2012. Befreiung vom Überfluss: Auf dem Weg in die Postwachstumsökonomie. München: Oekom.

Pentzold, Christian. 2015. Praxistheoretische Prinzipien, Traditionen und Perspektiven kulturalistischer Kommunikations- und Medienforschung. Medien und Kommunikationswissenschaft 63 (2): 229-245.

Portwood-Stacer, Laura. 2013. Media Refusal and Conspicuous Non-Consumption: The Performative and Political Dimensions of Facebook Abstention. New Media and Society 15 (7): 1041-1057.

Pufé, Iris. 2014. Nachhaltigkeit. 2., überarbeitete und erweiterte Auflage. Konstanz: UVK.

Raworth, Kate. 2017. Doughnut Economics: 7 Ways to Think Like a 21st Century Economist. Vermont: Chelsea Green.

Reckwitz, Andreas. 2003. Grundelemente einer Theorie sozialer Praktiken: Eine sozialtheoretische Perspektive. Zeitschrift für Soziologie 32 (4): 282-301.

Reißig, Rolf. 2009. Gesellschafts-Transformation im 21. Jahrhundert: Ein neues Konzept sozialen Wandels. Wiesbaden: VS.

Roitsch, Cindy. 2020. Kommunikative Grenzziehung: Herausforderungen und Praktiken junger Menschen in einer vielgestaltigen Medienumgebung. Wiesbaden: Springer VS. 
Schneidewind, Uwe. 2018. Die Große Transformation: Eine Einführung in die Kunst gesellschaftlichen Wandels. Frankfurt am Main: S. Fischer.

Schuler, Marcus. 2020. Rekord in der Corona-Krise Netflix boomt. tagesschau.de vom 22. April, https://www.tagesschau.de/wirtschaft/netflix-quartalszahlencoronavirus-103.html. Zugegriffen: 14. April 2021.

Sühlmann-Faul, Felix. 2019. Streaming heizt unserem Planeten ein: Die ökologischen Auswirkungen von Videostreaming. In: Was Bits und Bäume verbindet. Digitalisierung nachhaltig gestalten, herausgegeben von Anja Höfner und Vivian Frick, 32-33. München: Oekom.

van Dijk, José, und Thomas Poell. 2015. Social Media and the Transformation of Public Space. Social Media and Society 1 (2): 1-5.

van der Velden, Maja. 2018. ICT and Sustainability: Looking Beyond the Anthropocene. In: This Changes Everything - ICT and Climate Change: What Can We Do? herausgegeben von David Kreps, Charles Ess, Louise Leenen und Kai Kimppa, 166-180. New York: Springer.

Velkova, Julia. 2016. Data That Warms: Waste Heat, Infrastructural Convergence and the Computation Traffic Commodity. Big Data \& Society 3 (2): 1-10.

Vereinte Nationen. 2015. Resolution der Generalversammlung, verabschiedet am 1. September 2015. Entwurf des Ergebnisdokuments des Gipfeltreffens der Vereinten Nationen zur Verabschiedung der Post-2015-Entwicklungsagenda. https://www.un.org/Depts/german/gv-69/band3/ar69315.pdf. Zugegriffen: 14. April 2021.

Wilke, Jürgen. 2015. Theorien des Medienwandels: Versuch einer typologischen Systematisierung. In: Theorien des Medienwandels, herausgegeben von Susanne Kinnebrock, Christian Schwarzenegger und Thomas Birkner, 29-50. Köln: Herbert von Halem.

Winseck, Dwayne, und Dal Jong Yin. 2011. The Political Economies of Media: The Transformation of the Global Media Industries. London: Bloomsbury Academic.

WBGU. 2011. Welt im Wandel. Gesellschaftsvertrag für eine Große Transformation. Hauptgutachten des Wissenschaftlichen Beirats der Bundesregierung Globale Umweltveränderungen. Bonn. https://www.wbgu.de/fileadmin/user_upload/wbgu/publikationen/hauptgutachten/hg2011/pdf/wbgu_jg2011.pdf.

Wittmayer, Julia, und Katharina Hölscher. 2017. Transformationsforschung: Definitionen, Ansätze, Methoden. Dessau-Roßlau: Umweltbundesamt.

WCED. 1987. Report of the World Commission on Environment and Development: Our Common Future. http://www.un-documents.net/wced-ocf.htm. Zugegriffen: 14. April 2021.

Young, Carrie, und Katherine McComas. 2016. Media's Role in Enhancing Sustainable Development in Zambia. Mass Communication and Society 19 (5): 626-649. Yu, Jinglei, Eric Williams, und Ju Meiting. 2010. Analysis of Material and Energy Consumption of Mobile Phones in China. Energy Policy 38: 4135-4141.

\section{Open Access}

Dieser Beitrag erscheint unter der Creative-Commons-Lizenz CC BY-ND 3.0 DE: https://creativecommons.org/licenses/by-nd/3.0/de/. 